\title{
THE BABY FRIENDLY HOSPITAL INITIATIVE: A CASE STUDY FROM NSW
}

\section{Joy Heads}

Royal Hospital for Women

South Eastern Sydney/Illawarra Area Health Service

An important determinant of the initiation of breastfeeding and of its continuation in the first year of an infant's life is the mother's experience in hospital (see 'Factors affecting breastfeeding practices: applying a conceptual framework' by Hector et al in this issue).

In recognition of the importance of the hospital experience in determining future breastfeeding behaviour, the United Nations Children's Fund (UNICEF) and the World Health Organization (WHO) developed in 1991 the Baby Friendly Hospital Initiative.

This article introduces the Baby Friendly Hospital Initiative and its introduction to Australia and describes the case study of the Royal Hospital for Women in Sydney, which is accredited as a Baby Friendly hospital.

\section{THE BABY FRIENDLY HOSPITAL INITIATIVE}

The Baby Friendly Hospital Initiative aims to give every baby the best start in life by ensuring a health care environment where breastfeeding is endorsed as the norm. Baby Friendly status is accredited to maternity units that pass a rigorous inspection and meet the 'Ten steps to successful breastfeeding' (Table 1). The evidence base for each of these steps was confirmed by a review in 1998 and by

TABLE 1

\section{THE TEN STEPS TO SUCCESSFUL BREASTFEEDING}

Every facility providing maternity services and care for newborn infants should:

1. Have a written breastfeeding policy that is routinely communicated to all staff.

2. Train all health care staff in skills necessary to implement this policy.

3. Inform all pregnant women about the benefits and management of breastfeeding.

4. Help mothers initiate breastfeeding within a half-hour of birth.

5. Show mothers how to breastfeed and how to maintain lactation even if they should be separated from their infants.

6. Give newborn infants no food or drink other than breastmilk, unless medically indicated.

7. Practice rooming-in - allow mothers and infants to remain together -24 hours a day.

8. Encourage breastfeeding on demand.

9. Give no artificial teats or pacifiers (also called dummies or soothers) to breastfeeding infants.

10. Foster the establishment of breastfeeding support groups and refer mothers to them on discharge from the hospital or clinic.

Source: The Baby Friendly Hospital Initiative (Australia). Available at www.bfhi.org.au/text/bfhi_ten_steps.html. Accessed 15 June 2005. more recent, systematic reviews, meta-analyses and other studies. ${ }^{1-6}$ A number of recent studies endorse the findings that the Baby Friendly Hospital Initiative increases the rates of initiation and duration of breastfeeding. This evidence originates from diverse countries such as Scotland, the United Kingdom, Republic of Belarus, the United States, Switzerland, and Brazil. ${ }^{7-16}$ Paediatric hospitals and community health services are also included in the Baby Friendly Initiative and are required to meet best practice guidelines that are tailored to their functions (Tables 2 and 3).

In order to be accredited, hospitals are required to have written evidence-based breastfeeding policies and staff training programs and to provide comprehensive information to all women booked to deliver their infants at the hospital. ${ }^{17} \mathrm{~A}$ Baby Friendly accredited hospital ensures that practices such as unnecessary separation of mother and baby and inappropriate supplementation with formula do not occur and that, on discharge, information about community support for breastfeeding is given to all mothers.

International experience indicates that Baby Friendly accreditation is a quality tool that enables health authorities, in both developed and developing countries, to monitor and evaluate their efforts to support improved breastfeeding practices through their health facilities. ${ }^{18-20}$

\section{THE BABY FRIENDLY HOSPITAL INITIATIVE IN AUSTRALIA}

Since 1995, the Australian College of Midwives Incorporated has facilitated the initiative in Australia. The college holds corporate governance over the body, BabyFriendly Hospital Initiative in Australia, which is, in turn, supported by state and territory committees and a project officer. In 2002 the college received a two-year grant from the Australian Commonwealth Department of Health and Ageing to support the initiative in Australia.

It is desirable that all hospitals in Australia become Baby Friendly for optimal support of breastfeeding. The most recent data indicates that there are currently 51 hospitals or facilities that are Baby Friendly accredited in Australia, representing approximately 18 per cent of all maternity units (Table 4).

\section{BABY FRIENDLY HOSPITAL INITIATIVE IN NSW}

In NSW, there are two maternity units that are currently Baby Friendly accredited (the Royal Hospital for Women and Queanbeyan Hospital). The Royal Hospital for Women was the first tertiary referral teaching hospital in NSW to achieve Baby Friendly status (which occurred in 1999) and is due for its second three-yearly re-accreditation in 2005. 


\section{BREASTFEEDING IN PAEDIATRIC UNITS GUIDANCE FOR GOOD PRACTICE}

Breastfeeding is the healthiest way that a woman can feed her baby, because it provides important health benefits to both her and her child. This is why all health care professionals working in the paediatric environment should actively encourage women to breastfeed their babies. The following is a list of measures which paediatric units could adopt to help achieve this aim.

1. Have a written breastfeeding policy that is routinely communicated to all health care staff and provide people with training to acquire the skills necessary to implement this policy.

2. Provide mothers with an environment and facilities which meet their needs for privacy, information and appropriate nutrition

3. Support mothers in their choice of feeding method - and assist in the establishment and maintenance of breastfeeding.

4. Provide parents with written and verbal information about the benefits of breastfeeding and breastmilk.

5. Use alternative techniques conducive to breastfeeding if a baby is unable to feed at the breast.

6. Give no bottles or dummies to breastfeeding babies unless medically indicated and with parents' permission.

7. Provide facilities that allow mothers and babies to be together 24 hours a day in order to promote breastfeeding on demand.

8. Plan all nursing and medical care to minimise disturbance to the breastfeeding relationship.

9. Provide mothers with a dedicated facility that is appropriately furnished with well-maintained and sterilised equipment for the safe expression and storage of breastmilk.

10. Provide parents with information about breastfeeding support groups during admission and on discharge from hospital.

Source: The UNICEF UK Baby Friendly Initiative. Breastfeeding in paediatric units-guidance for good practice. London, Royal College of Nursing.

\section{TABLE 3}

THE SEVEN POINT PLAN FOR THE PROTECTION, PROMOTION AND SUPPORT OF BREASTFEEDING IN COMMUNITY HEALTH CARE SETTINGS

All providers of community health care should:

1. Have a written breastfeeding policy that is routinely communicated to all healthcare staff

2. Train all staff involved in the care of mothers and babies in the skills necessary to implement the policy

3. Inform all pregnant women about the benefits and management of breastfeeding

4. Support mothers to initiate and maintain breastfeeding

5. Encourage exclusive and continued breastfeeding, with appropriately-timed introduction of complementary foods

6. Provide a welcoming atmosphere for breastfeeding families

7. Promote co-operation between healthcare staff, breastfeeding support groups and the local community

Source: The UNICEF UK Baby Friendly Intitiative.

\section{A CASE STUDY}

For the Royal Hospital for Women, achieving and maintaining Baby Friendly status is inextricably linked to building a strong breastfeeding culture at the hospital and the regu-
BABY FRIENDLY HOSPITAL INITIATIVE ACCREDITED HOSPITALS IN AUSTRALIA, BY STATE, 21/2/05

\begin{tabular}{|lcc|}
\hline State/ & No. of hospitals & \% of maternity units \\
Territory & \multicolumn{2}{l}{} \\
\hline ACT & 2 & 66.7 \\
NSW & 2 & 4.0 \\
NT & 3 & 50.0 \\
Qld & 6 & 12.7 \\
SA & 8 & 19.5 \\
Tas & 4 & 50.0 \\
Vic & 23 & 20.5 \\
WA & 2 & 3.0 \\
Total & 51 & 17.5 \\
\hline Source: & Baby Friendly Hospital Initiative Australia e-bulletin. \\
& Feb 2005 (4) At www.bfhi.org.au/text/bulletins/21_2_ \\
& 05.htm & \\
\hline
\end{tabular}

lar monitoring of prevailing practices and breastfeeding outcomes. The principles of the Baby Friendly Hospital Initiative place the mother and baby, as an inseparable unit, at the centre of all care. This underpins the hospital's philosophy and mission statement, which is 'Providing care in partnership with women'.

In 1995 the Executive Director and clinicians decided to work towards Baby Friendly accreditation, prior to the hospital's move to the new Randwick site in 1997. Therefore, achieving future Baby Friendly status was considered in the planning and layout of the new postnatal wards. Visitors are still surprised when they do not find a nursery full of babies to 'view' but instead an empty 'arrivals lounge' and babies rooming-in. Wall clocks are no longer in all rooms (to support 'demand feeding') and breastfeeding pictures from many cultures adorn the walls in all areas of the hospital.

The Baby Friendly Hospital Initiative's tenth step encourages maternity facilities to 'foster the establishment of breastfeeding support groups and refer mothers to them on discharge from the hospital'. The hospital has done this by having a small unit on the postnatal ward where women can be seen post-discharge for complex breastfeeding issues. Results of a 2002 sample of 50 women found that 72 per cent of women were breastfeeding at six months. ${ }^{21}$ This is considerably higher than the proportion breastfeeding at six months for the NSW population (42.5 per cent). Considering that these women were referred for complex breastfeeding issues, this suggests that this service is an effective model of postnatal care for maintaining breastfeeding rates.

Attitudinal change among staff continues to prove the greatest challenge to those wishing to seek Baby Friendly accreditation. Much of the educational focus at the Royal Hospital for Women during the preparatory time was to reassure the staff that there would not be a dramatic change in practices. Changes in practice had gradually occurred 
over the previous decade with the introduction of evidencebased care.

Working in a Baby Friendly hospital means many things. For example, new staff know in advance the policies, practices and routines that are expected. It means that staff are supported in their on-going breastfeeding education by regular in-service training. It also means financial support for external education from the Royal Hospital for Women's Nursing and Midwifery Education Fund.

At the Royal Hospital for Women, practices affecting breastfeeding outcomes are monitored by random sampling twice a year. These practices include:

- post-birth uninterrupted skin-to-skin contact and timely initiation of first feed

- dummy use

- formula supplementation of breastfeeding babies and assessment of the stated medical reasons

- maternal consent for formula supplementation and dummy use

- early assistance for mothers of pre-term babies with expressing their breast milk.

The Royal Hospital for Women found that implementing the initiative was helped by:

- support from hospital administrators and first line managers

- the hospital Executive's support for the overall initiative

- building and maintaining a breastfeeding culture in the hospital

- training staff to ensure that they understand the initiative and understand that it does not involve a substantial additional workload

- ongoing review of the evidence base for the hospital's routine practices

- development of the Breastfeeding Support Unit, again with the support of hospital administrators and staff

- regular monitoring of current practices and outcomes.

The Royal Hospital for Women has a tradition of striving for the best in health care and consequently Baby Friendly accreditation is viewed as an integral part of our hospital. Comments in support of the Baby Friendly Hospital Initiative are numerous. For example, feedback in the Baby Friendly Hospital Initiative's Assessors report (2002) included 'All mothers interviewed were also very articulate and confident in their breastfeeding knowledge. A number of women commented that they were surprised that they had not received any conflicting advice, as their friends had warned them that this may happen'.22 Such positive comments inspire staff to protect and maintain Royal Hospital for Women's Baby Friendly status.

\section{WHERE TO FROM HERE IN NSW AND AUSTRALIA?}

Despite 10 years of experience with the Baby Friendly Hospital Initiative in Australia, its uptake by hospitals remains limited. The reasons for this are complex. Breastfeeding and postnatal care are often not the main focus for many midwives and maternity units, whose central philosophy is quality prenatal care and birthing options. Therefore, the Baby Friendly Initiative remains largely driven by very motivated individuals. Shorter length of hospital stay for newly delivered women mean that women are often discharged before they have learnt the basic skills of newborn feeding and sleeping norms. The Baby Friendly Hospital Initiative is not a high priority activity for area health services and as a result few resources are directed to devising and implementing strategies to improve breastfeeding behaviour. Greater support from policy makers and managers at the state, area health service and institutional levels would help the widespread implementation of the initiative and, ultimately, improve breastfeeding outcomes.

Why are there such large differences in uptake of the initiative across Australia? Differences can be explained by differences in the priorities of local branches of the Australian College of Midwives Incorporated in each state and territory. For hospitals to be appropriately supported to achieve and maintain Baby Friendly Hospital Initiative accreditation their state branch of the Australian College of Midwives Incorporated must see the initiative as part of their core business, in equal concert with antenatal care and birthing options, and must commit resources to the initiative. Endorsement of the initiative is integral to the upcoming NSW breastfeeding policy (as described by Macoun in 'The NSW Health breastfeeding project' in this issue of the Bulletin).

Achieving Baby Friendly status in a facility requires a commitment from all staff to ensure that the hospital's culture sees breastfeeding as the normal way of feeding babies and takes into consideration interventions that support or undermine that basic concept.

To increase the number of maternity units achieving Baby Friendly Hospital Initiative accreditation in Australia, we require:

- policy and financial support from national and state health authorities to encourage routine implementation of this evidence-based strategy to increase breastfeeding rates

- the use of 'breastfeeding at discharge' and 'breastfeeding duration' rates, plus the application of the 'ten steps' as benchmarks for achievement by national and state health authorities

- state branches of the Australian College of Midwives Incorporated to see Baby Friendly hospital accreditation 
as their core business and to commit further resources to the initiative

- support for services progressing to Baby Friendly hospital to help them identify their specific barriers and to overcome these barriers, and

- dissemination of successful models of Baby Friendly hospitals.

More information can be found at the Australian Baby Friendly Hospital Initiative website: www.bfhi.org.au.

\section{REFERENCES}

1. World Health Organization. Division of Child Health and Development. Evidence for the ten steps to successful breastfeeding. Geneva, Switzerland: World Health Organisation; 1998.

2. Sikorski J, Renfrew MJ, Pindoria S, Wade A. Support for breastfeeding mothers. Cochrane Database Syst Rev 2002; 1 (most recent update 21 January 2002).

3. Anderson GC, Moore E, Hepworth J, Bergman N. Early skin-to-skin contact for mothers and their healthy newborn infants. Cochrane Database Syst Rev, 2003; 2 (most recent update 22 April 2003).

4. Donnelly A, Snowden HM, Renfrew MJ, Woolridge MW. Commercial hospital discharge packs for breastfeeding women. Cochrane Database Syst Rev 2000; 2 (most recent update 24 April 2000).

5. Goldstein Ferber S, Makhoul IR. The effect of skin-toskin contact (kangaroo care) shortly after birth on the neurobehavioral responses of the term newborn: a randomised controlled trial, Pediatrics. 2004; 113(4):858-865.

6. Hector D, King L, Webb K. State of food and nutrition in NSW series. Overview of recent reviews to promote and support breastfeeding in NSW. Sydney: NSW Department of Health 2004

7. Kramer MS, Chalmers, Hodnett ED, Sevkovskaya Z. et al. Promotion of breastfeeding intervention trial (PROBIT): a randomized trial in the Republic of Belarus. JAMA. 2001; 285: 413-420.

8. Phillip BL, Merewood A, Miller LW, et al. Baby Friendly Initiative improves breastfeeding initiation rates in a US hospital setting. Pediatrics. 2001; 108(3): 677-681.

9. Cattaneo A, Buzzetti R. Effects on rates of breast-feeding of training for the Baby Friendly Hospital Initiative. $\mathrm{Br} \mathrm{Med} \mathrm{J}$ 2001; 323:1658-1662.
10. Merewood A, Phillip BL, Chawla N. The Baby Friendly Hospital Initiative increases breastfeeding rates in a US neonatal intensive care unit. J Hum Lact 2003;19(2): 166-171.

11. Merten S, Ackermann-Liebrich U. Exclusive breastfeeding rates and associated factors in Swiss Baby Friendly Hospitals. J. Hum Lact 2004; 20(1): 9-17.

12. UNICEF UK Baby Friendly Initiative. Baby Friendly hospitals show strong increase in breastfeeding rates. Baby Friendly News July 2000; 6.

13. Braun MLG, Guigliana ERJ, Mattos Soares ME, Guigliani C, de Oliveira AP, Danelon CMM. Evaluation of the impact of the Baby Friendly hospital initiative on rates of breastfeeding. Am J Public Health 2003; 93(8): 1277-1279.

14. Philipp BL, Malone KL, Cimo S, Merewood A. Sustained breastfeeding rates at a US Baby Friendly Hospital. Pediatrics 2003; 112(3): 234-236.

15. Do Nascimento MB, Issler $H$. Breastfeeding the premature infant: experience of a Baby Friendly hospital in Brazil. J Human Lact 2005; 21(1): 47-52.

16. Broadfoot M, Britten J, Tappin DM, MacKenzie JM. The Baby Friendly Hospital Initiative and breastfeeding rates in Scotland. Arch Dis Child Fetal Neonatal Ed 2005; 90(2): 114-116.

17. Labarere J, Castell M, Fourny M et al. A training program on exclusive breastfeeding in maternity wards. Int J Gynecol Obstet 2003; 83:77-84.

18. Hillenbrand KM, Larsen PG. Effects of an educational intervention about breastfeeding knowledge, confidence and behaviors of pediatric resident physicians. Pediatrics 2002; 110(5): e59.

19. Radford A, Southall D P. Successful application of the Baby Friendly Hospital Initiative contains lessons that must be applied to the control of formula feeding in hospitals in industrialised countries [Commentary]. Pediatrics 2001; 108(3): 766-768.

20. Tappin DM. Breastfeeding rates are increasing in Scotland. Health Bulletin 2001; 59:102-107.

21. Heads J, Miles C. A cool place in a hot spot: A BFHI tertiary referral hospital's answer to the $10^{\text {th }}$ Step. Conference handbook: milk. mammals and marsupials: an international perspective. Conference of the International Lactation Consultants Association. (Abstract) pp185-189; 2003.

22. Report from Baby Friendly Hospital Initiative RHW assessment team 27-28 March 2002. Received April 2002. 용 